University of Wollongong

Research Online

Faculty of Engineering and Information

Faculty of Engineering and Information

Sciences - Papers: Part A

Sciences

$1-1-2013$

Free-standing nanocomposites with high conductivity and extensibility

Kyoung-Yong Chun

Hanyang University

Shi Hyeong Kim

Hanyang University

Min Kyoon Shin

Hanyang University

Youn Tae Kim

Chosun University, Korea

Geoffrey M. Spinks

University of Wollongong, gspinks@uow.edu.au

See next page for additional authors

Follow this and additional works at: https://ro.uow.edu.au/eispapers

Part of the Engineering Commons, and the Science and Technology Studies Commons

Research Online is the open access institutional repository for the University of Wollongong. For further information contact the UOW Library: research-pubs@uow.edu.au 


\title{
Free-standing nanocomposites with high conductivity and extensibility
}

\begin{abstract}
The prospect of electronic circuits that are stretchable and bendable promises tantalizing applications such as skin-like electronics, roll-up displays, conformable sensors and actuators, and lightweight solar cells. The preparation of highly conductive and highly extensible materials remains a challenge for mass production applications, such as free-standing films or printable composite inks. Here we present a nanocomposite material consisting of carbon nanotubes, ionic liquid, silver nanoparticles, and polystyrene- polyisoprene-polystyrene having a high electrical conductivity of $3700 \mathrm{~S} \mathrm{~cm}-1$ that can be stretched to $288 \%$ without permanent damage. The material is prepared as a concentrated dispersion suitable for simple processing into free-standing films. For the unstrained state, the measured thermal conductivity for the electronically conducting elastomeric nanoparticle film is relatively high and shows a non-metallic temperature dependence consistent with phonon transport, while the temperature dependence of electrical resistivity is metallic. We connect an electric fan to a DC power supply using the films to demonstrate their utility as an elastomeric electronic interconnect. The huge strain sensitivity and the very low temperature coefficient of resistivity suggest their applicability as strain sensors, including those that operate directly to control motors and other devices. (c) 2013 IOP Publishing Ltd.
\end{abstract}

\section{Keywords}

extensibility, standing, free, nanocomposites, high, conductivity

Disciplines

Engineering | Science and Technology Studies

\section{Publication Details}

Chun, K., Kim, S. Hyeong., Shin, M. Kyoon., Kim, Y. Tae., Spinks, G. M., Aliev, A. E., Baughman, R. H. \& Kim, S. Jeong. (2013). Free-standing nanocomposites with high conductivity and extensibility. Nanotechnology, 24 (16), 1-9.

\section{Authors}

Kyoung-Yong Chun, Shi Hyeong Kim, Min Kyoon Shin, Youn Tae Kim, Geoffrey M. Spinks, Ali E. Aliev, Ray H. Baughman, and Seon Jeong Kim 


\title{
Free-standing nanocomposites with high conductivity and extensibility
}

Running heads: Extensible free-standing nanocomposite conductive films

Kyoung-Yong Chun ${ }^{1}$, Shi Hyeong Kim¹, Min Kyoon Shin ${ }^{1}$, Youn Tae Kim², Geoffrey M. Spinks², Ali E. Aliev $^{3}$, Ray H. Baughman ${ }^{3}$ and Seon Jeong Kim ${ }^{*}$

${ }^{1}$ Center for Bio-Artificial Muscle and Department of Biomedical Engineering, Hanyang University, Seoul, 133791, Korea

${ }^{2}$ IT Fusion Technology Research Center and Department of IT Fusion Technology, Chosun University, Gwangju, 501-759, Korea

${ }^{3}$ ARC Centre of Excellence for Electromaterials Science, Intelligent Polymer Research Institute, University of Wollongong, Wollongong, NSW, 2522, Australia

${ }^{4}$ The Alan G. MacDiarmid NanoTech Institute, University of Texas at Dallas, Richardson, TX 75083, USA

*E-mail: sjk@hanyang.ac.kr

\begin{abstract}
The prospect of electronic circuits that are stretchable and bendable promises tantalizing applications such as skin-like electronics, roll-up displays, conformable sensors and actuators, and lightweight solar cells. The preparation of highly conductive and highly extensible materials is still a remaining challenge amenable to mass production such as free-standing film or printable composite ink. Here we present a nanocomposite material consisting of carbon nanotubes, ionic liquid, silver nanoparticles, and polystyrene-polyisoprene-polystyrene having a high electrical conductivity of $3700 \mathrm{~S} \mathrm{~cm}^{-1}$ that can be stretched to $288 \%$ without permanent damage. The material is prepared as a concentrated dispersion suitable for simple processing into free-standing films. For the unstrained
\end{abstract}


state, the measured thermal conductivity for the electronically conducting elastomeric nanoparticle film is relatively high and shows a non-metallic temperature dependence consistent with phonon transport, while the temperature dependence of electrical resistivity is metallic. We connect an electric fan to a DC power supply using the films to demonstrate their utility as an elastomeric electronic interconnect. The giant strain sensitivity and the very low temperature coefficient of resistivity suggest applicability for strain sensors, including those that operate directly to control motors and other devices.

Keywords: Conductivity, stretchability, composite film, carbon nanotubes, silver nanoparticles

\section{Introduction}

Three main strategies have been explored to overcome the inherent incompatibility of high electronic conductivity and high reversible extensibility in materials. Firstly, thin film silicon-based electronics have been laminated within polymer sheets so that they lie close to the neutral axis during sheet bending [1]. This strategy ensures that the silicon strain is low and damage avoided, but the method is only successful for low radius bends and is not suitable for stretching. Silicon and other metal components have been geometrically modified to induce flexibility and high strain elasticity in tension [2-9]. Corrugated sheets or perforated mesh-like films allows for large deformations while maintaining a low strain within the material elements. Finally, composite materials containing conductive particles embedded within an elastomeric matrix have also been extensively investigated [10-18]. The most successful systems retain a percolated conductive network to high strains.

The property requirements for stretchable electronics depend very much on the application. The ability to sustain strains of up to 30\% for displays [8] and 100\% for actuators [17] without permanent mechanical or electrical degradation has been reported. For electronic interconnects, the desire is to produce the highest conductivity possible, so as to reduce resistance losses and maximize the current capacity of the circuit. A resistivity that is insensitive to strain is desirable for electronic interconnects. For mechanical sensors, however, a change in resistance with strain is needed. Finally, for cost minimization the materials need to be suitable for mass production, with printing-based assembly a 
popular option.

Recent reports [10-13] set the benchmark in terms of a material that combines high conductivity, high strain elasticity and processability. Chun et al [13] report a composite material consisting of multi-walled carbon nanotubes (MWNTs) and silver flakes prepared as a solution with an ionic liquid and a supporting film-forming polymer. The composite film had a conductivity of $3,100 \mathrm{~S} \mathrm{~cm}^{-1}$ at zero strain that decreased to $60 \mathrm{~S} \mathrm{~cm}^{-1}$ at $30 \%$ strain before curing and hot rolling at elevated temperature. These composite films had excellent electrical conductivity, but low extensibility themselves. Similar systems that use single-walled carbon nanotubes (SWNTs) give lower conductivities: $57 \mathrm{~S} \mathrm{~cm}^{-1}$ at $38 \%$ strain [10]; $102 \mathrm{~S} \mathrm{~cm}^{-1}$ at $29 \%$ strain [11]; $50 \mathrm{~S} \mathrm{~cm}^{-1}$ at $30 \%$ strain [14]; and $125 \mathrm{~S} \mathrm{~cm}^{-1}$ at zero strain [19] for a coated textile.

Here we present elastomeric nanocomposite films which have a high-electrical conductivity of $\sim 3,700 \mathrm{~S} \mathrm{~cm}^{-1}$ at $0 \%$ strain and $\sim 50 \mathrm{~S} \mathrm{~cm}^{-1}$ at $100 \%$ strain. The films were prepared by casting a dispersion of MWNTs, an ionic liquid, and Ag nanoparticles in a polystyrene-polyisoprenepolystyrene (SIS) matrix. The same solutions were successfully screen printed onto plastic substrates. The utility of the nanocomposite film as an elastomerically deformable current conductor was demonstrated by using this film as a link in the power circuit for the fan of a personal computer: even when stretched $30 \%$ the link provided $8 \mathrm{~V}$ to the fan for 300 hours without causing severe performance degradation of the fan.

\section{Experimental section}

\subsection{Preparation of the nanocomposite film}

MWNTs with an outer diameter of $10 \mathrm{~nm}$ (Nanocyl, $60 \mathrm{mg}$ ) were mixed and ground with $150 \mathrm{mg}$ ionic liquid (1-butyl-4-methylpyridinium tetrafluoroborate) using a pestle and mortar for 30 min to yield a black gel [10]. The MWNT gel (210 mg) was blended with a SIS copolymer in toluene, and sonicated at room temperature (200 W, 3 min). Silver nanoparticles (Nanostructured \& Amorphous Materials Inc. spherical type, with an average size of $35 \mathrm{~nm}$ ) were added into the MWNT-SIS solution and sonicated (600 W, $20 \mathrm{~min})$. To make homogeneous composite solution, 30 vol\% of toluene versus SIS 
copolymer solution was added and sonicated (600 W, 5 min). Finally, the Ag-MWNT-SIS nanocomposite (AMSN) film was obtained by slip casting on a slide glass or a polymer film and drying at $70^{\circ} \mathrm{C}$ for $2 \mathrm{~h}$. Free-standing AMSN film was obtained by peeling the dried AMSN film out of the substrate. Typical thickness range of the AMSN film was $80 \sim 100 \mu \mathrm{m}$.

\subsection{Measurement of electrical and thermal conductivity}

The electrical conductivity of thefilm was measured using a two-point probe (4-wiring system). An ohm meter (Delta United Instrument Co., Ltd. DU-5111) was used for the resistance measurement of the film. The strain was applied using a house-built device as shown in figure S1. The thermal conductivity and thermal diffusivity of CNT composite were measured using the self heating 3-omega method. The conventional schematic diagram of $3 \omega$ measurement setup is shown in figure S2. The detail method was descirbed in the supplementary data. The Agilent 33220A Functional Generator which has spurious $3 \omega$ signal below of $0.1 \mu \mathrm{V}$ was used in high impedance mode as a current source. The Stanford Research System SR830 DSP Lock-In Amplifier can digitally pick up the third harmonic signal across the specimen, which contains valuable thermal transport data. The sample was suspended in vacuum (Janis Research VPF-475 CRYOSTAT, 0.1 mbar, with liquid nitrogen cooling) on four gold deposited copper bars. Silver-epoxy glue (H-20) deposited on outward electrodes was cured at $100^{\circ} \mathrm{C}$ for $30 \mathrm{~min}$.

\subsection{The operation of PC fan using an elastomeric electronic interconnect}

A standard 3W fan used for PC ventilation was used for this demonstration. The PC fan was operated at voltages between about 3 and 8 V using a DC power supply (Testlink, Korea, VS-350Q). Strain was applied to the elastomeric film using a home-built device. Fan rotation rate was measured by counting the reflection of laser beam from a metal film that on attached to the fan. Current-voltage characteristic of PC fan was measured up to $30 \%$ strain of the connecting elastomeric film using a current meter (Keithley 2400). The power consumption $(P)$ of conducting elastomeric film was calculated by 


$$
P=I^{2} R,
$$

where $I$ is current, $L$ is the film length, $R$ is the electrical resistance of the film.

\subsection{Characterization}

The morphology of the composite film was investigated by FE-SEM (Hitachi, S4800) and EDS (Horiba, EMAX). The mechanical properties of the rubber film were measured using an Instron 5900 testing machine of Instron Corporation.

\section{Results and discussion}

\subsection{Preparation of AMSN films}

Figure 1 schematically illustrates the preparation of AMSN free-standing film. In brief, MWNTs (average diameter: $10 \mathrm{~nm}$ ) were mixed with a pyridinium-based ionic liquid (1-butyl-4methylpyridinium tetrafluoroborate), which has an ionic conductivity of $1.854 \mathrm{mS} \mathrm{cm}^{-1}$ [20] and is known to effectively disperse nanotubes [21] without causing substantial damage to the nanotubes [22]. Complete gelation of MWNTs and ionic liquid occurred when a 2.5 weight ratio of ionic liquid versus MWNTs were mixed using a mortar and pestle (figure 1a), which is the weight ratio used for all experiments. The MWNT gel was further mixed with SIS copolymer in toluene, and then sonicated (figure 1b).

SIS copolymer was chosen as the matrix due to its excellent mechanical properties as rubber (the elongation to break and tensile strength were $1480 \%$ and $8.75 \mathrm{MPa}$, respectively (supplementary data, figure S3). Also, use of $\pi-\pi$ interaction of aromatic rings in polymers is known to be effective for both binding carbon nanotubes (CNTs) and dispersing them without damaging the $\mathrm{sp}^{2}$ structure and thereby interrupting CNT conjugation [23-25]. Therefore, aromatic rings on the polystyrene block should be effective for stacking the SIS molecules onto the CNT surface via $\pi-\pi$ interaction; dispersing the CNTs; and generating strong physical interactions between CNTs and SIS polymer causing improved stretchability. In the next processing step (figure 1c) Ag nanoparticles were added to the MWNTs-SIS solution, and then the mixture was sonicated. Finally, an AMSNfilm with an average thickness of 20 
$\mu \mathrm{m}$ was prepared by slip casting and drying at $70^{\circ} \mathrm{C}$ (figure 2a). After drying, the AMSNfilm was easily peeled out of a glass slide. Figure $2 \mathrm{~b}$ shows that the AMSN dispersion can also be successfully screen printed on a polyethylene terephthalate film at room temperature. No delamination occurred during flexing of the printed substrate.

The AMSN films showed a complex arrangement of Ag nanoparticles and MWNTs embedded in the SIS copolymer matrix (figure 2 and supplementary data, figure S4). The cross sectional image of the film obtained by field-emission scanning electron microscopy (SEM) show the dispersed MWNTs in the polymer matrix (figures 2c and 2d). Clustered Ag nanoparticles (35 nm in average diameter) are distributed as shown in the inset of figure 2c. The magnified SEM image shows that Ag nanoparticles clustered in the range of 100 to $200 \mathrm{~nm}$. Also, it is seen that MWNTs surround and conjugate with Ag nano-cluster in the SIS matrix (figure 2d). The combination of high electrical conductivity and high elastomeric deformability for the nanocomposite sheets might relate in part to the bridging of $\mathrm{Ag}$ nanoparticles by carbon nanotubes. The SEM micrograph of figure 2d suggests that the MWNTs form a cocoon-like layer about an array of Ag nanoparticles. This "cocooning” when done for a continuous 3D network of Ag nanoparticles should facilitate Ag percolation and nanoscale separation between rubber-rich, Ag-poor regions and Ag-rich areas, thereby enabling the Ag-poor regions of the composite to provide reversible elastomeric behavior. Whatever the correct explanation, the present results, which build on previous important results for Ag flakes [13], provides important compositions for possible commercial application, and challenging materials for more complete theoretical understanding.

\subsection{Electrical conductivity and stretchability of the AMSN films}

Figure 3a shows the electrical conductivity of the AMSN films as a function of the weight and volume percentages of Ag nanoparticles. To examine the effect of MWNTs concentration on the resistance change of the films, the concentration of MWNTs in SIS solution was changed. Various amounts of MWNTs were mixed and ground with ionic liquid (2.5 times vs. MWNTs) using a pestle and mortar for $30 \mathrm{~min}$. MWNT gels were added into SIS solution and sonicated (600 W, $20 \mathrm{~min}$ ) to make 
homogeneous MWNTs-SIS composite solution. Finally, the MWNT-SIS films were homogeneously prepared up to 2 weight \% (wt\%), but crack was generated in the films at over $3.5 \mathrm{wt} \%$ (supplementary data, figure S5). Also, the resistance of MWNT-SIS films rapidly decreased with increase of the MWNT concentration up to $2 \mathrm{wt} \%$, and the decrease of film resistance with increasing nanotube concentration was small above 2.7 wt\% of MWNTs (supplementary data, figure S6). Therefore, the amounts of components except Ag nanoparticles were kept constant (MWNTs, 60 mg; ionic liquid, $150 \mathrm{mg}$; SIS, 2.6 g).

In order to investigate the percolation of Ag nanoparticles in the MWNT-SIS composites, the electrical conductivity was measured according to the amount of Ag nanoparticles. The dimension of the composite films were $20 \times 7 \times(0.08 \sim 0.1) \mathrm{mm}$. As shown in the figure 3a, the electrical conductivity of the AMSN films rapidly increased when the volume of Ag nanoparticles in the dried film was above 29 volume \% (vol\%), which is approximately the experimental percolation threshold. At the peak, the electrical conductivity reached an average value of $\sim 3,700 \mathrm{~S} \mathrm{~cm}^{-1}$ at $40 \mathrm{vol} \%$. At this conductivity of the film, the composition of all components was 1:2.6:45.4:51 (MWNTs:ionic liquid:SIS:Ag). Addition of Ag nanoparticles above $40 \mathrm{vol} \%$ in the dried films decreased electrical conductivity of the films. At 46 vol\% of Ag nanoparticles (corresponding to $55 \mathrm{wt} \%$ of $\mathrm{Ag}$ in the precursor solution) the surface of the cast films started to crack due to phase separation.

The theoretical prediction for the electrical conductivity of the AMSN films was obtained using a power-law relationship and three-dimensional percolation theory $[13,26]$. Briefly, the assumed powerlaw relationship is given by

$$
\sigma=\sigma_{0}\left(V_{\mathrm{f}}-V_{\mathrm{c}}\right)^{s}
$$

where $\sigma$ is the electrical conductivity of the composite, $\sigma_{0}$ is the electrical conductivity of the conductive filler $\left(\sigma_{0}=86,000 \mathrm{~S} \mathrm{~cm}^{-1}\right.$ for bulk Ag, which were assumed to be the main conducting elements), $V_{\mathrm{f}}$ is the volume fraction of thefiller, $V_{\mathrm{c}}$ is the critical volume fraction at the percolation threshold, and $s$ is the exponent in the power law indicating networks dimensionality. The theoretically 
obtained value of $s$ for a 3D percolation was 1.36. The Ag nanofiller was modeled as 3D randomly distributed isotropic nanoparticles, and the percolation threshold was calculated using the average interparticle distance model [26]. Power-law fitting using an experimentally derived percolation threshold of 29 vol\% after the drying process, which is equivalent to $34 \mathrm{wt} \%$ in the initial mixture, showed good agreement with the experimental data on conductivity as a function of Ag loading (figure 3a) and the 26 vol\% average interparticle distance model. No attempt was made to fit the conductivity data above 40 vol\% due to the cracking and phase separation of Ag nanoparticles that limited electrical conductivity.

The role of a minor concentration of carbon nanotubes (typically $1 \mathrm{wt} \%$ ) in enabling the combination of high conductivity and high elasticity is important, but complex. The percolation thresholds of pure CNTs and pure Ag nanoparticles in a SIS polymer matrix were about 1 and 37 wt\%, respectively. In general, the metallic continuum in a dielectric matrix can be achieved with high Ag loadings in the range of 30 80 wt\% $[27,28]$. Above the percolation threshold, the adjacent Ag fillers coalesce inducing the formation of larger nanoclusters [27]. The nanoclusters increase the connectivity of fillers and result in the enhancement of electrical conductivity due to efficient electron tunneling [29]. In the present work, the percolation threshold of the AMSN of Ag in the MWNT-containing composite was $37 \mathrm{wt} \%$. Experimentally, it was confirmed that the film made with $37 \mathrm{wt} \% \mathrm{Ag}$ nanoparticles without CNTs had a conductivity of only $\sim 75 \mathrm{~S} \mathrm{~cm}^{-1}$.

The strain sensitivity of the electrical conductivity of the free-standing AMSNfilms was measured to various strain limits using a two-point probe system (figure 3b). Because elastomeric SIS copolymer has excellent mechanical properties such as the elongation to break (1480\%) and tensile strength (8.75 MPa) (supplementary data, figure S3) the conductive films consisting of MWNTs and Ag nanoparticles can be sustained even at quite large strain. The two-point probe electrical conductivity system used to measure electrical conductivity as a function of strain is described (Supporting Information, figure S5). An average conductivity of $\sim 3,700 \mathrm{~S} \mathrm{~cm}^{-1}$ was obtained at $0 \%$ strain for all 4 samples prepared with an Ag nanoparticle concentration of $51 \mathrm{wt} \%$ corresponding to 40 vol\% and SIS solution of either $10 \mathrm{wt} \%$ or $15 \mathrm{wt} \%$ in toluene. The conductivities decreased with 
increasing strain, with the samples prepared at $15 \mathrm{wt} \%$ decreasing more slowly than films prepared from the $10 \mathrm{wt} \%$ solution. The highest conductivity at $30 \%$ strain was $1,500 \mathrm{~S} \mathrm{~cm}^{-1}$ and at $100 \%$ strain was $\sim 50 \mathrm{~S} \mathrm{~cm}^{-1}$. These conductivities are amongst the highest reported at equivalent strains $[10,11,13]$ and only exceeded by as system composed of a silver paste coated onto a corrugated rubber substrate [8], which is a method that cannot be used for ordinary printing and results in substrate bound material that cannot be strained to above $60 \%$ without causing failure. A pure Ag film without CNTs had a conductivity of $\sim 75 \mathrm{~S} \mathrm{~cm}^{-1}$. Cyclic tests on the AMSN films to either 10 or 30\% maximum strains and up to 300 cycles were performed, which indicates reversibly strain-dependent conductivity after the first few "break-in cycles”(figure 3c). Usually, in the case of elastomeric conductive film, the structural deformation was observed during the stretching cycles [7]. During the durability test, disconnection between materials in the composites can be created and inhibiting the electrical pathway. Therefore, it is considered that stretchability of samples can be enhanced by introducing perforation which can induce relaxation in the stress concentration and increase elasticity under tensile load [30]. Elastic rubber substrates also increase the stretchability of a conductivefilm that is embedded in the substrate or attached to the substrate, while maintaining its electrical conductivity $[1,2,4,7,9,19]$. In this study, however, the free-standing AMSN film without the aid of rubber substrates was compared with other control materials because of high electrical conductivity and elongation of AMSN film itself. During stretch and recovery of samples, the electrical conductivity of AMSN film was $~ 1,600 \mathrm{~S}$ $\mathrm{cm}^{-1}$ at $30 \%$ strain after 300 cycles. This value even if no any substrate is the highest among other reports. For example, the electrical conductivity of the textile coated with SWNT ink was $\sim 125 \mathrm{~S} \mathrm{~cm}^{-1}$. The value of $R / R_{0}$ decreased to $\sim 0.825$ until the strain reached $140 \%$, and then increased as the strain increased further ( 0.95 at $229 \%$ strain) [19]. The electrical conductivity of poly(ethylene oxide) gel/Ag composites on a polydimethylsiloxane (PDMS) elastomer substrate was $18,000 \mathrm{~S} \mathrm{~cm}^{-1}$ at $40 \%$ strain. The weight fraction of Ag nanoparticles was, however, very high in the value of $60 \%$ [16]. Also, silver micro electrode on a substrate showed $118 \mathrm{~S} \mathrm{~cm}^{-1}$ at $10 \%$ strain [5]. However, these materials have no printability required for large-scaled electronic systems.

Printable composites with electrical conductivity of $57 \mathrm{~S} \mathrm{~cm}^{-1}$ at $38 \%$ strain have been reported 
[10]. The stretchability was then further improved at the maximum electrical conductivity (which fell to $6 \mathrm{~S} \mathrm{~cm}^{-1}$ at $134 \%$ strain) by perforating the nanotube film and then coating it with PDMS substrate. Jet milling has also been used to improve the performance of nanotubefilms to a maximum electrical conductivity of $102 \mathrm{~S} \mathrm{~cm}^{-1}$ at $29 \%$ strain and $9.7 \mathrm{~S} \mathrm{~cm}^{-1}$ at $118 \%$ strain [11]. Very recently, Chun et al obtained the conductive films with $5170 \mathrm{~S} \mathrm{~cm}^{-1}$ at $0 \%$ strain and $706 \mathrm{~S} \mathrm{~cm}^{-1}$ at $30 \%$ strain by using hotrolling method and elastomeric substrate [13]. However, all of the films without substrate were fractured around 35\% strain. The AMSN films have much higher electrical conductivity in the factor of 2.8 at $0 \%$ strain and 5 at 30\% strain in comparison with previous their results. Most stretchable and conductive composite materials have been incoporated into the polymer substrate such as PDMS in order to improve stretchability $[4,6,7]$. However, we here fabricated highly conducting, easily fabricated elastomeric films without need for a substrate and other treatments such as hot rolling and annealing methods.

The highest achieved electrical conductivity $\left(3,700 \mathrm{~S} \mathrm{~cm}^{-1}\right)$ was substantially reduced during initial cycling used to obtain reversible dependence of electrical conductivity as a function of strain (figure 3c). However, the final reversible electrical conductivity in $30 \%$ strained state is still sufficiently high for use as an elastomeric interconnect. This reversible electrical conductivity is approximately exponential in applied strain, which indicates that free-standing AMSN films can be useful as strain sensors. The average coefficient of reversible fractional resistance change $\left(R-R_{0} / R_{0}\right)$ between 0 and $30 \%$ is a giant $270 \%$ per $\%$ strain. In comparison with other reports, the SWNTs/silicone rubber composite films retain their conductivity as $18 \mathrm{~S} \mathrm{~cm}^{-1}$ after 20th cycle test up to $300 \%$ strain [14]. The maximum conductivity of this film is $63 \mathrm{~S} \mathrm{~cm}^{-1}$ at $0 \%$ strain after acid treatment of the film.

\subsection{Temperature dependence of resistivity and thermal conductivity for AMSN films}

Considering the giant dependence of electrical conductivity on strain and the large positive thermal expansion that can be expected for the elastomer matrix, the temperature dependence of the AMSN film resistance $(R)$ is surprisingly linear in temperature and small in the investigated wide 
temperature range $80 \mathrm{~K}$ and $350 \mathrm{~K}$ (figure 4a). It means that in electrical conductivity of CNT/Ag composite the contribution of $\mathrm{Ag}$ nanoparticles is dominant in whole measured region because the resistance linearly increases with increasing temperature in many metals and metal-polymer complexes [31-33]. The coefficient $\mathrm{d} R / R \mathrm{~d} T \sim 3.3 \times 10^{-3} \mathrm{~K}^{-1}$ is close to that of bulk high purity silver (3.8 $\times 10^{-3} \mathrm{~K}^{-1}$ and $4 \times 10^{-3}$ to $2.5 \times 10^{-3} \mathrm{~K}^{-1}$ for Ag nanowires having diameters between 15 and $200 \mathrm{~nm}$ [34]. The combination of this small dependence of electrical on temperature, the exponential dependence of electrical conductivity of strain, and electrical conductivity reversibility up to $30 \%$ strain swuggests that the AMSN films are particularly attractive for application as mechanical strain sensors. In contrast with the metallic temperature dependence of electrical conductivity, the AMSN films shows the non-metallic temperature dependence of thermal conductivity (figure $4 \mathrm{~b}$ ) that one would expect for phonon transport. The thermal conductivity at $300 \mathrm{~K}$ is $8.7 \mathrm{~W} / \mathrm{Km}$, which is above 40 times that of the SIS polymer matrix.

The electrical performance of the AMSNfilm as an interconnect material was demonstrated by operating a PC fan, as schematically illustrated in figure 5a. The fan operated successfully at DC voltages from $3 \mathrm{~V}$ (figure 5b) and fan speed was not greatly affected by stretching the film to $30 \%$ strain when the applied voltage exceeded $5 \mathrm{~V}$ (figures 5c, 5d, and 5e). Fan speed was substantially reduced at strains over 50\% (figure 5c) - fan operation can be shown in the movie (supplementary data, movie S1). The power consumption of the AMSN film connection to the PC fan was $\sim 30 \mu \mathrm{W}$ at $6 \mathrm{~V}$ total voltage, compared with $3 \mathrm{~W}$ for the fan itself, and the circuit current-voltage response of the AMSN film at $0 \%$ strain is totally identical to copper foil (figure 5f). The current was constant when the film was stretched to $30 \%$ strain. Also, the current-voltage characteristics were highly reversible when the strain was released. The fan was operated at $8 \mathrm{~V}$ for $300 \mathrm{~h}$ at $30 \%$ strain without any degradation of the AMSN film.

\section{Conclusions}

In conclusion, we present free-standing elastomeric nanocomposites with ultrahigh conductivity that can operate in high current circuits. The nanocomposites were prepared by mixing MWNTs, an ionic 
liquid, and Ag nanoparticles in polystyrene-polyisoprene-polystyrene copolymer matrix. The electrical conductivity of the film reached $\sim 3,700 \mathrm{~S} \mathrm{~cm}^{-1}$ at $0 \%$ strain and $\sim 50 \mathrm{~S} \mathrm{~cm}^{-1}$ at $100 \%$ strain in the free standing films without substrate and without perforation or corrugation. A PC fan circuit was operated using a conducting link provided by the composite films at various DC voltage at up to $70 \%$ strain. The AMSN film provided comparable performance to copper sheet having about the same dimensions, but could also maintain the revolution rate of the PC fan at 3V to $30 \%$ strain. In addition, the current carrying capability of the AMSN film itself were 6 and $3 \mathrm{~A} / \mathrm{mm}^{2}$ at zero and $30 \%$ strain, respectively. Also, the observed combination of a very small dependence of conductivity on temperature with an exponential dependence on strain suggests the application of AMSN films as strain sensors. This composite film can be also apply to diverse areas requiring highly deformable interconnects, such as construction of high-power energy harvesting, joints of robot's arm, and large-scaled stretchable display and actuators.

\section{Acknowledgements}

This work was supported by Creative Research Initiative Center for Bio-Artificial Muscle of the Ministry of Education, Science and Technology (MEST) and the MEST-US Air Force Cooperation Program (Grant No.2011-00178) in Korea and Air Force Grant AOARD-10-4067, Air Force Office of Scientific Research grant FA9550-09-1-0537, and Robert A. Welch Foundation grant AT-0029 in the USA and the Australian Research Council through the Centre of Excellence program.

\section{References}

[1] Suo Z, Ma E Y, Gleskova S and Wagner S 1999 Appl. Phys. Lett. 741177

[2] Kim D H, Ahn J H, Choi W M, Kim H S, Kim T H, Song J, Huang Y Y, Liu Z and Rogers J A 2008 Science 320507

[3] Sun D M, Timmermans M Y, Tian Y, Nasibulin A G, Kauppinen E I, Kishimoto S, Mizutani T and 
Ohno Y, 2011 Nature Nanotech. 6156

[4] Sun Y G, Choi W M, Jiang H Q, Huang Y G Y and Rogers J A 2006 Nature Nanotech.1 201

[5] Ahn B Y, Duoss E B, Motala M J, Guo X Y, Park S I, Xiong Y J, Yoon J, Nuzzo R G, Rogers J A and Lewis J A 2009 Science 3231590

[6] D Y Khang, Jiang H Q, Huang Y and Rogers J A 2006 Science 311208

[7] Yamada T, Hayamizu Y, Yamamoto Y, Yomogdia Y, Izadi-Najafabadi A, Futaba D N and Hata K 2011 Nature Nanotech. 6296

[8] D C Hyun, Park M, Park C, Kim B, Xia Y, Hur J H, Kim J M, Park J J and Jeong U 2011 Adv. Mater. 232946

[9] Wang C, Zheng W, Yue Z, Too C O and Wallace G G 2011 Adv. Mater. 232946

[10] Sekitani T, Noguchi Y, Hata K, Fukushima T, Aida T and Someya T 2008 Science 3211468

[11] Sekitani T, Nakajima H, Maeda H, Fukushima T, Aida T, Hata K and Someya T 2009 Nature Mater. 8494

[12] Sekitani T, Nakamiya M, Noguchi Y, Nakano S, Kato Y, Sakurai T and Someya T 2007 Nature Mater. 6413

[13] Chun K Y, Oh Y, Rho J, Ahn J H, Kim Y J, Choi H R and Baik S 2010 Nature Nanotech. 5853

[14] Kim T A, Kim H S, Lee S S and Park M 2012 Carbon 50444

[15] Shang S, Zeng W and Tao X 2011 J. Mat. Chem. 217274

[16] Wang X, Hu H, Shen Y, Zhou X and Zheng Z 2011 Adv. Mater. 233090

[17] Pelrine R, Kornbluh R, Pei Q B and Joseph J 2000 Science 287836

[18] Kim K H, Vural M and Islam M F 2011 Adv. Mater. 232865

[19] Hu L B, Pasta M, La Mantia F, Cui L F, Jeong S, Deshazer H D, Choi J W, Han S M and Cui Y 2010 Nano Lett. 10708

[20] Bandrés I, Montaño D F, Gascón I, Cea P and Lafuente C J 2010 Electrochimica Acta 552252

[21] Price B K, Hudson J L, Tour M and Green J M 2005 J. Am. Chem. Soc. 12714867

[22] Jarosik A, Krajewski S R, Lewandowski A and Radzimski P 2006 J. Mol. Liq. 12343

[23] Foroutan M and Nasrabadi A T 2010 J. Phys. Chem. B 1145320 
[24] Yang Q, Shuai L and Pan X 2008 Biomacromolecules 93422

[25] Yuan W, Che J and Chan-Park M B 2011 Chem. Mater. 234149

[26] Li J and Kim J 2007 Comp. Sci. Tech. 672114

[27] Wei H and Eilers H 2008 Thin Solid Films 517575

[28] Jiguet S, Bertsch A, Hofmann H and Renaud P 2004 Adv. Eng. Mater. 6719

[29] Kiesow A, Morris J E, Radehaus C and Heilmann A 2003 J. Appl. Phys. 946988

[30] Shigley J E and Mischeke C R 2001 Mechanical Engineering Design (New-York: McGraw-Hill) 130

[31] Nassary M M 2005 J. Alloy. Comp. 39821

[32] Kavlak S, Kodolbas A O, Can H K, Guner A and Rzaev Z M O 2004 Adv. Poly. Tech. 23222

[33] Zhang J Q, Feng L H, Cai W, Zheng J G, Cai Y P, Li B, Wu L L and Shao Y 2002 Thin Solid Films 414113

[34] Bid A, Bora A and Raychaudhuri A K 2006 Phys. Rev. B 74035426 


\section{Lists of Figure}

Figure 1. Schematic illustration of the preparation of Ag-MWNT-SIS nanocomposite (AMSN) films. (a) MWNT gel prepared by mixing and grinding MWNTs with ionic liquid (1-butyl-4methylpyridinium tetrafluoroborate). (b) MWNT-SIS solution prepared by sonication of MWNT gel in a SIS copolymer solution (200 W for $3 \mathrm{~min}$ ). SIS copolymer has benzene rings on the polystyrene blocks which can be easily interaction with carbon nanotubes. (c) Ag nanoparticles were mixed with MWNT-SIS composite solution by sonication ( $600 \mathrm{~W}$ for $5 \mathrm{~min}$ ). The average size of the $\mathrm{Ag}$ nanoparticles was $35 \mathrm{~nm}$. AMSN composite film was finally obtained by slip casting and drying.

Figure 2. Images of AMSN films. (a) Slip casting an AMSIfilm (scale bar: $1 \mathrm{~cm}$ ). (b) A $\mathrm{n}$ AMSN film coating formed by screen printing an AMSN solution on a transparent polyethylene terephthalate film (scale bar: $1 \mathrm{~cm}$ ). (c) SEM image of cross section of the AfISAN The inset is a cross sectional image showing the $\sim 20 \mu \mathrm{m}$ thickness of the AMSN film. (d) Magnification SEM image of the cross section of the AMSN film.

Figure 3. Electrical conductivity and stretchability of AMSNfilms. (a) Electrical conductivity of the AMSN film, composed of MWNTs (1 wt\%) and various concentrations of spherical Ag nanoparticles (0 60 wt\%). Percolation occurs $37 \mathrm{wt} \%$ loading of the Ag nanoparticles corresponding to 29 vol\% of Ag. The wine color line is a prediction based on a power-law relationship (equation (1) in main text) and three-dimensional percolation theory. The SEM images of AMSN films in the graph (labeled according to the wt\% of Ag in the precursor solution) indicate that the density of Ag nanoparticles is increasing according to the increase of the fraction of Ag nanoparticles. (b) Electrical conductivity of the AMSN films versus strain for four different fractions of Ag nanoparticles and both 10 and 15 wt\% SIS. (c) Electrical conductivity changes for free-standing AMSN films upon repeated cycles of stretching and strain release for both 10 and 30\% maximum strain. The additional data points provide comparisons with previous literature results: diamond, silver micro electrode at $10 \%$ strain [5]; square, 
super growth SWNT films under 25\% strain [10]; triangle, SWNT/silicone rubber composite electrode at $30 \%$ strain [14].

Figure 4. (a) Electrical resistance and (b) thermal conductivity as a function of temperature for a freestanding AMSN film. The thermal conductivities were measured using the 3-omega technique at fixed current $(I=1 \mathrm{~mA})$ and frequency $(f=0.1 \mathrm{~Hz})$. These AMSN files comprised $1 \mathrm{wt} \%$ of MWNTs, 2.6 wt $\%$ of ionic liquid, $45.4 \mathrm{wt} \%$ of the SIS polymer, and $51 \mathrm{wt} \%$ of Ag nanoparticles.

Figure 5. The operation of a PC fan using a stretched AMSN film as an electronic interconnect. (a) Schematic illustration of measurement configuration. (b) Images of static fan (top) and during fan operation at $5 \mathrm{~V}$ while the AMSN film is stretched by $30 \%$ (bottom). (c) Rotation rate of the PC fan as a function of elongation of the AMSN electronic interconnect for different applied voltages. (d) The revolution rate as a function of voltage for 30\% applied strain on the AMSN interconnect. Inset: The dependence of fan rotation rate on the strain applied to the AMSN film. For all applied voltages this applied maximum strain had little effect on fan rotation rate. (e) Fan rotation rate (for $5 \mathrm{~V}$ applied voltage) as the strain in the AMSN film is repeatedly cycled between $0 \%$ and $30 \%$. (f) Currentvoltage characteristics of PC fan measured during film stretch to $30 \%$ elongation and after release of strain to $0 \%$ elongation. As a control, the PC fan was operated using copper foil having approximately the same dimensions as the AMSN film at $0 \%$ strain. 


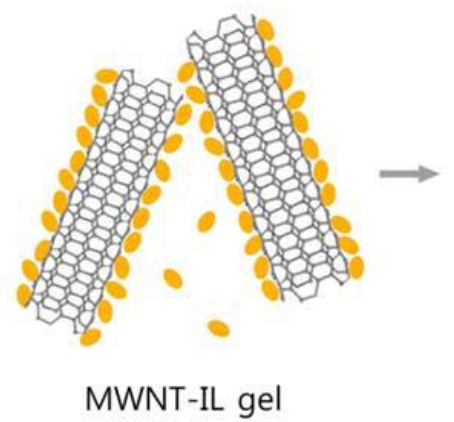

(a)

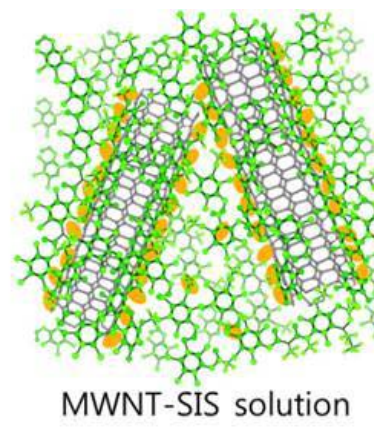

(b)

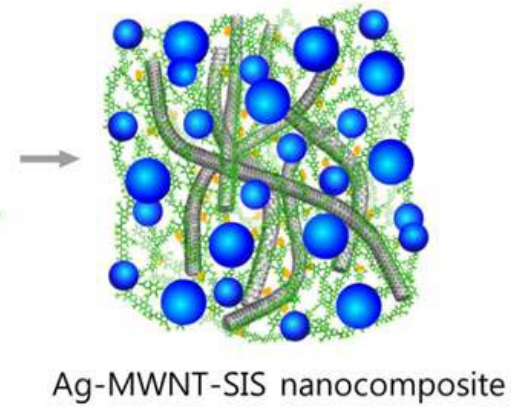

(c)

Figure 1 
(a)

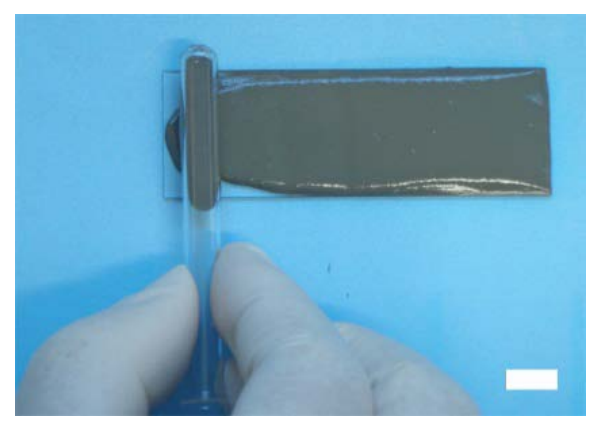

(c)

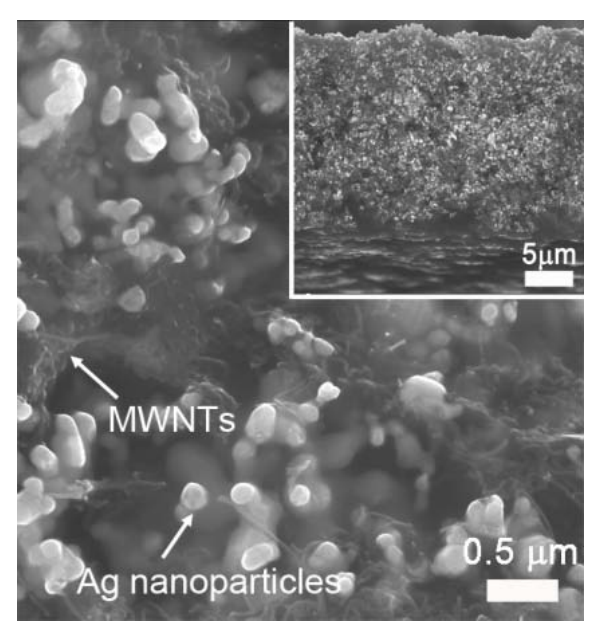

(b)

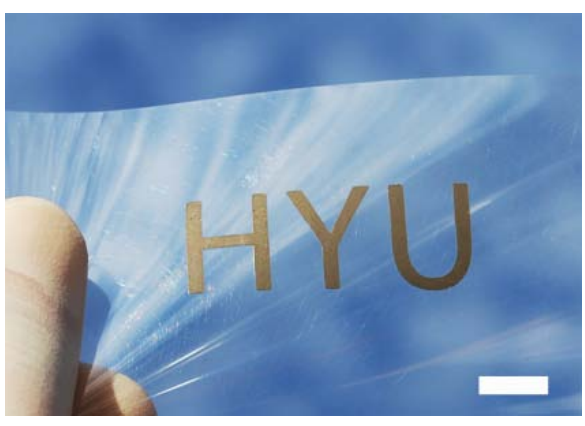

(d)

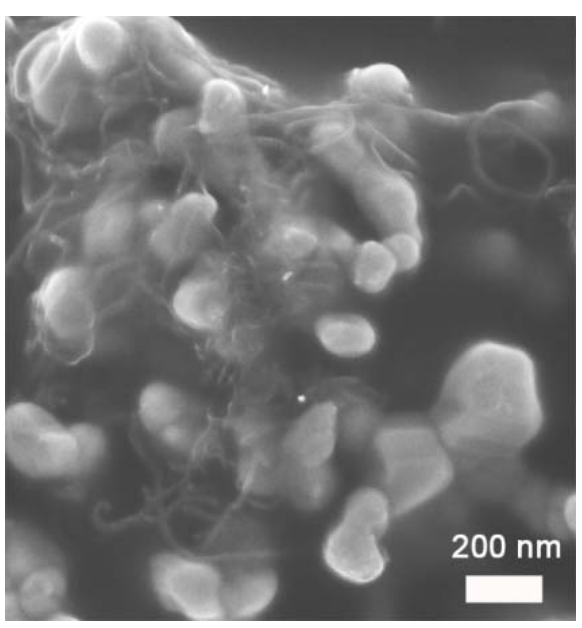

Figure 2 
(a)

Volume of Ag nanoparticles (\%)

after the drying process

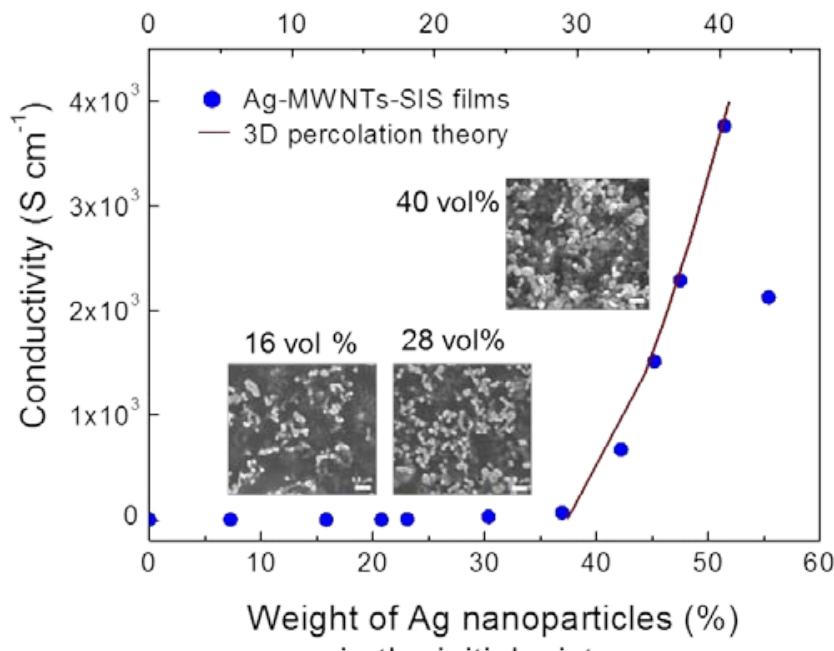

in the initial mixture

(b)

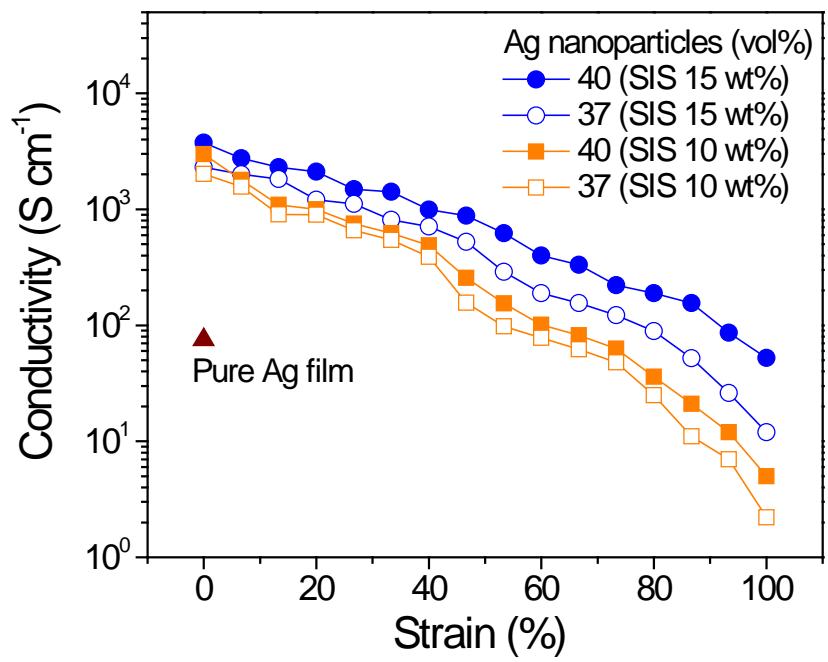

(c)

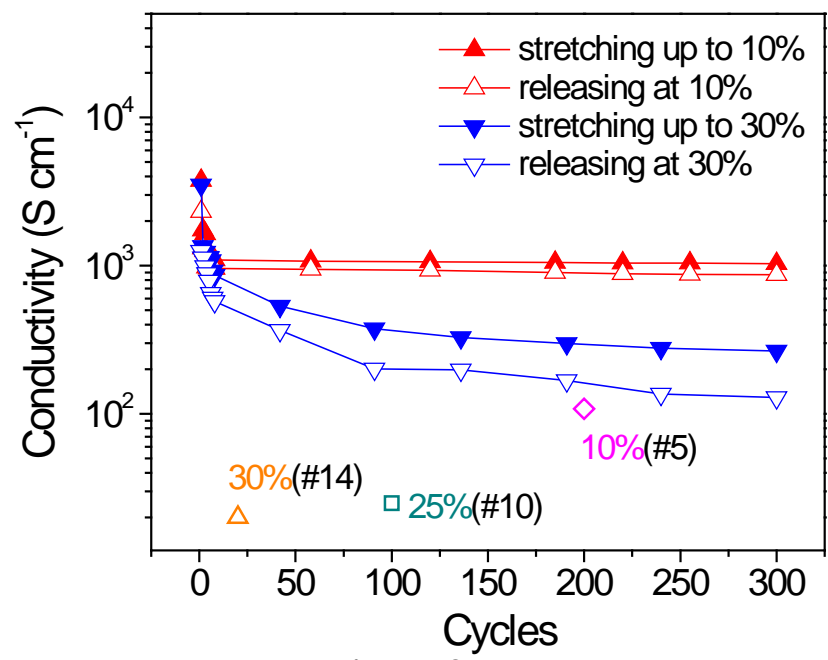

Figure 3 


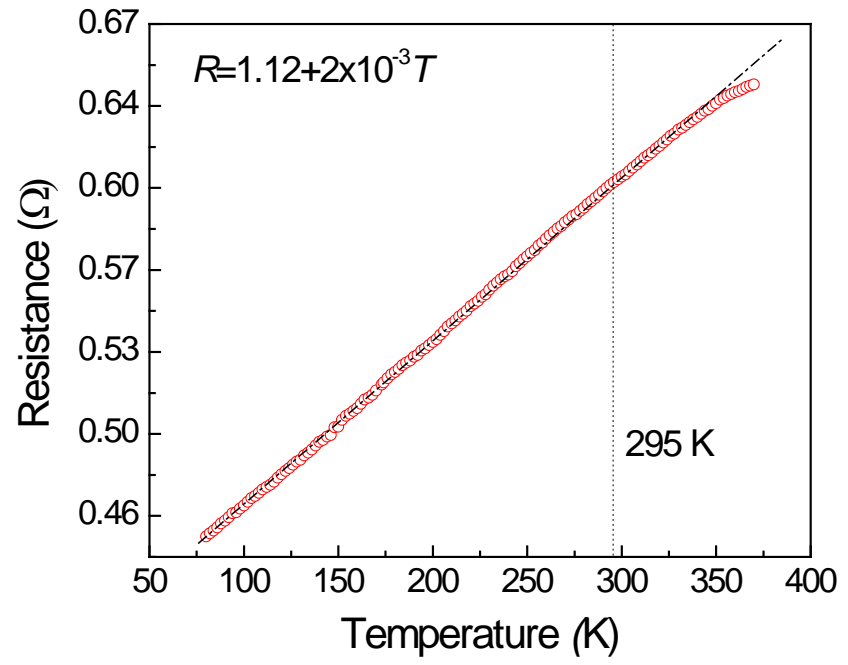

(a)

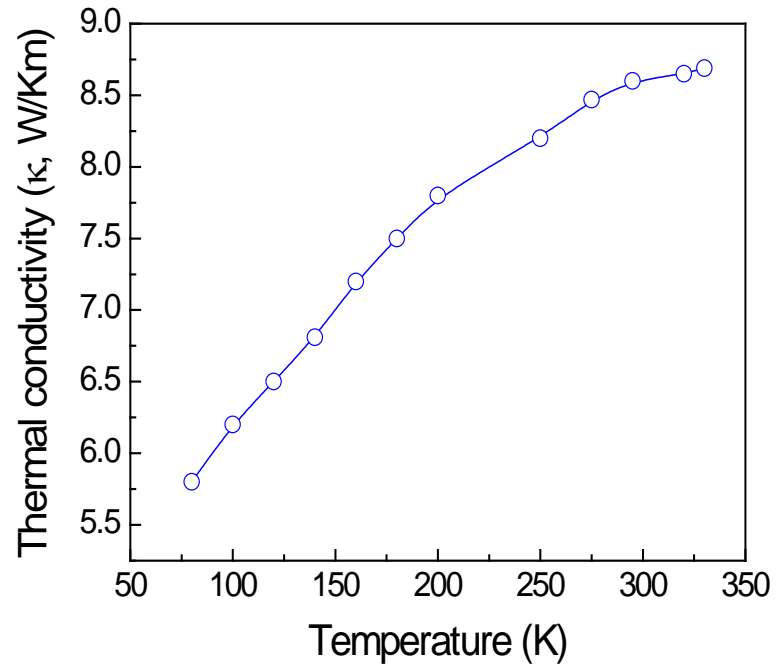

(b)

Figure 4 


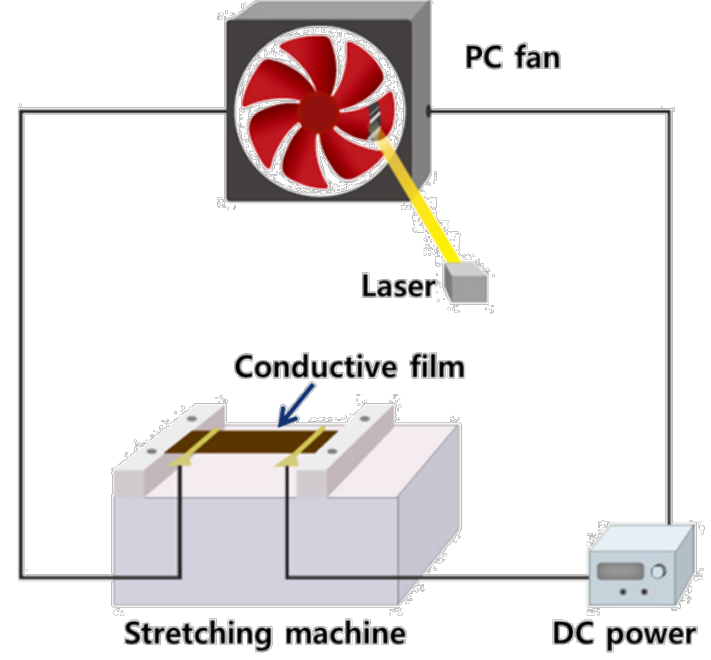

(a)

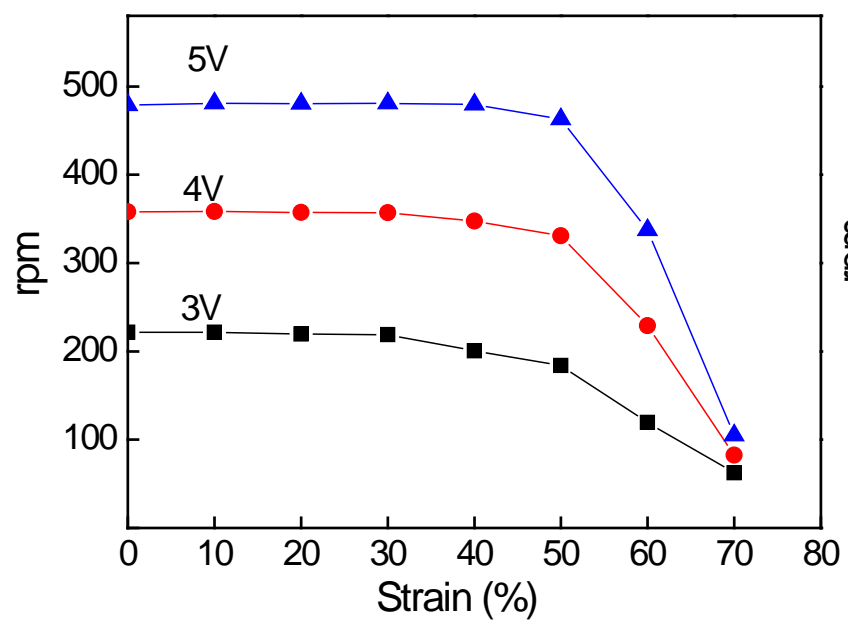

(c)

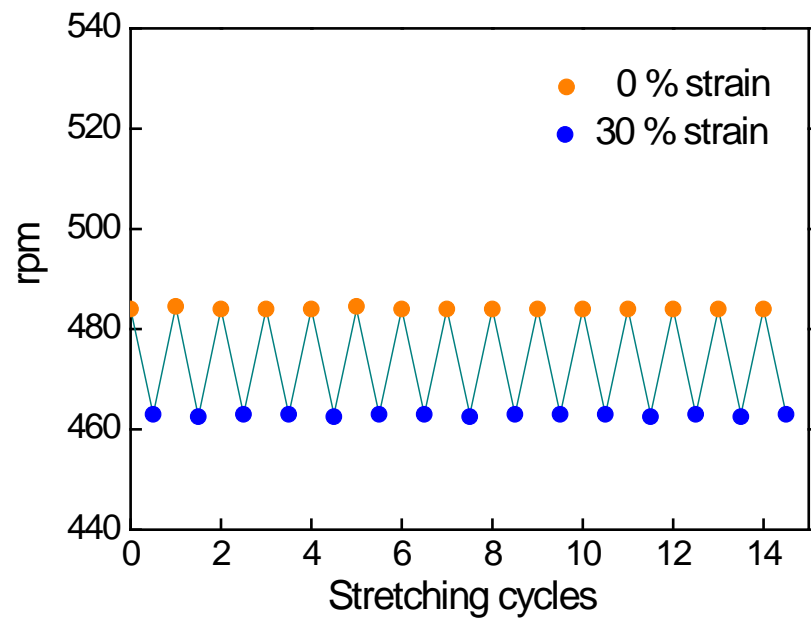

(e)

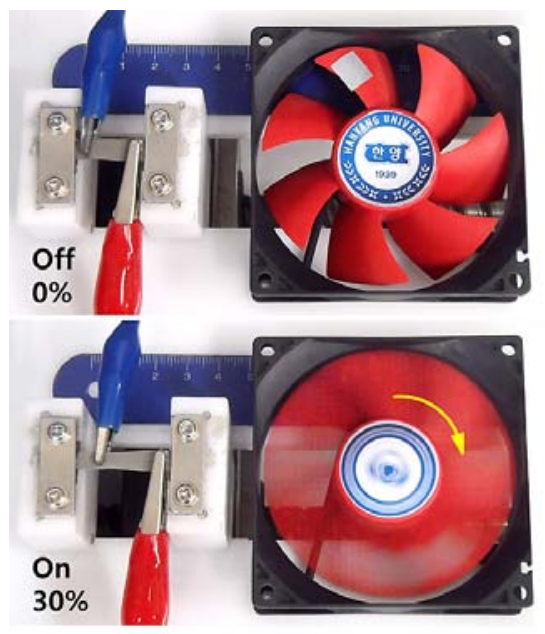

(b)

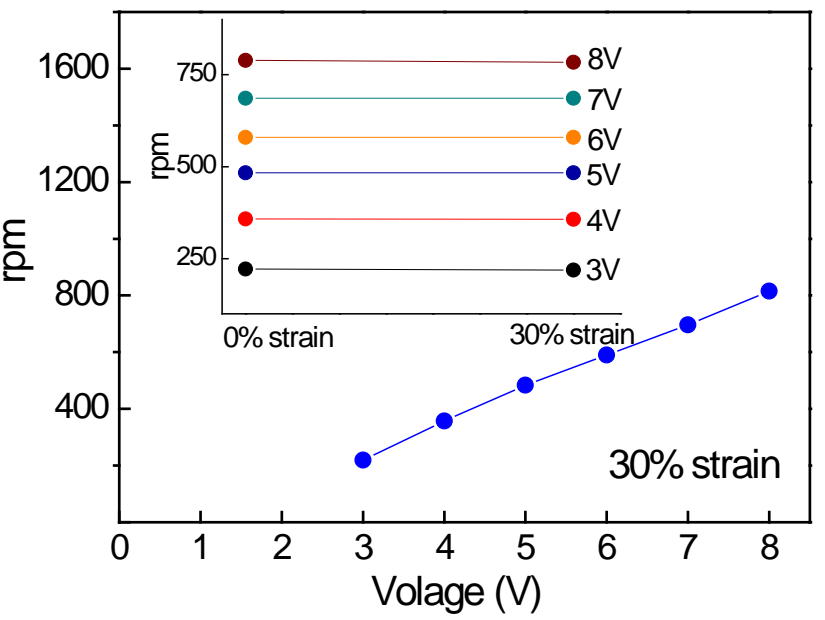

(d)

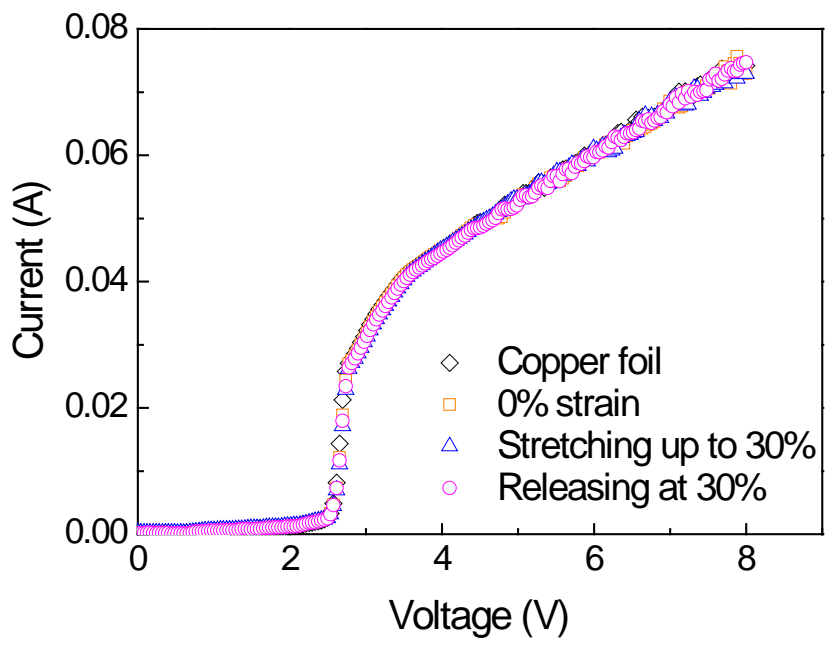

(f)

Figure 5 